
\title{
3 Research Square \\ Loss of Stat3 in Osteoblasts Impaired the Bone Remodeling in Inflammatory Microenvironment
}

\section{Jingyi Feng}

Sun Yat-Sen University https://orcid.org/0000-0001-5064-7484

\section{Zijing Huang}

Sun Yat-Sen University

\section{Laiting Chan}

Sun Yat-Sen University

Jiarui Lu

Sun Yat-Sen University

\section{Xin Feng}

Sun Yat-Sen University

Lizhen Lei

Sun Yat-Sen University

\section{Zhuwei Huang}

Sun Yat-Sen University

Lichieh Lin

Sun Yat-Sen University

\section{Yichen Yao}

Sun Yat-Sen University

Xiaolei Zhang ( $\nabla$ zhangxl35@mail.sysu.edu.cn)

Sun Yat-sen University https://orcid.org/0000-0003-2410-179X

\section{Research}

Keywords: Signal transducer and activator of transcription-3, osteoblasts, bone remodeling, inflammation, proliferation, migration

Posted Date: October 19th, 2021

DOI: https://doi.org/10.21203/rs.3.rs-966668/v1

License: (c) (i) This work is licensed under a Creative Commons Attribution 4.0 International License. Read Full License 


\section{Abstract}

Introduction: Oral diseases including periodontitis, periapical periodontitis, and peri-implantitis are characterized by inflammation and loss of alveolar bone. Signal transducer and activator of transcription 3 (Stat3) involved in bone formation and maintenance. This study aimed to investigate the effect of Stat3 on the osteoblast-mediated bone remodeling in the inflammatory lesion.

Methods: The conditional knockout of Stat3 in osteoblasts (Stat3 CKO) was generated via the Cre-loxP recombination system using Osterix-Cre (Osx-Cre) transgenic mice. The lipopolysaccharide (LPS)-induced calvarial bone inflammatory lesions were harvested at day 3, 7 and 14 for micro-CT scanning, histological staining, quantitative real-time PCR (qRT-PCR) and western blot to assess the bone remodeling. Osteoblasts were harvested from Stat3 CKO and wildtype mice and were cultured in vitro. In response to LPS-stimulation, the cells were subjected to alizarin red staining, qRT-PCR and western blot to examine the formation of calcium deposit, the expression of osteogenic markers (i.e. Runx2, OPN, COL1A1) and osteoclast-related markers (i.e. RANKL, OPG). The EdU and transwell assays were performed to assess the proliferation and migration of the cells.

Results: Compared with the wildtype control, a decrease of bone mass and an increase of osteolysis were found in the inflammatory lesions on Stat3 CKO mice. More osteoclastic-like cells, as well as an increased expression of $R A N K L$, were observed in the lesions on Stat3 CKO mice. Both mRNA and protein expressions of Stat3 and osteogenic markers in the lesions was significantly decreased on Stat3 CKO mice. After co-cultured with osteogenic medium, the Stat3-deficient osteoblasts were found with a significant decrease of calcium deposits and the expression of osteogenic markers, and with a significant increased expression of $R A N K L$, when compared with the control osteoblasts. The impaired ossification of Stat3-deficient osteoblasts was even more pronounced with the presence of LPS in vitro. According to the EdU and transwell assays, the most decrease of cell proliferation and migration was found in Stat3deficient osteoblasts in response to LPS.

Conclusions: Loss of Stat3 in osteoblasts impaired bone regeneration in inflammatory microenvironment, which might be resulted from the effect of Stat3 on the ossification, activation of osteoclast activationic signals, proliferation and migration of osteoblasts.

\section{Introduction}

The inflammatory oral bone diseases, including periapical periodontitis, periodontitis and peri-implantitis, are characterized by inflammation, resorption of alveolar bone and connective tissues [1, 2]. Accordingly, how to control the inflammatory destruction of bone tissue and to promote bone regeneration is an important clinic challenge and bench topic.

Osteoblasts and osteoclasts are regulated, in part, by many local factors including cytokines in the bone microenvironment. Janus kinase (JAK)-signal transducer and activator of transcription (Stat) pathway have been also found in bone cells [3]. The Stats consisting of Stat1, Stat2, Stat3, Stat4, Stat5a, Stat5 $\beta$ 
and Stat6, are an important family of intracellular transcription factors that mediate cell signal transduction with dual functions of transmitting cytoplasmic signals and initiating gene transcription in the nucleus [4]. Stat3 mediated by numerous growth factors, cytokines and oncoproteins, plays an important role in cell proliferation, survival, apoptosis, differentiation, transformation, cellular immunity and so on [5].

Among the seven Stats, Stat3 was reported to be the most relevant to bone disorders [6]. In human, the Job syndrome (also named as the high IgE syndrome) is caused by Stat3 mutation, which is an autosomal dominant multisystem disorder characterized by recurrent skin and lung infections, high serum IgE levels, and obvious craniofacial and skeletal features [7, 8]. Fractures, joint hyperextension and scoliosis were observed on Job syndrome patients [9]. Similarly, our previous study showed that the selective inactivation of mesenchymal progenitors-derived Stat3 in mice led to shortened limbs, multiple fractures and reduced bone mass due to inhibition of bone formation, suggesting an important role of Stat3 during bone development [10]. What's more, we also found that overexpression of Stat3 rescued the decreased osteoblast differentiation of Ror2-knockdown mBMSCs, indicating Stat3 could be the downstream molecule of Ror2 [11]. Stat3 can be found in osteoblasts, osteocytes, chondrocytes and osteoclasts [6]. Studies have shown that the depletion of Stat3 from osteoclasts caused a marked increase of trabecular bone mass in adult mice, in which the number of osteoclast was decreased without influencing the activity of osteoblasts [12]. Meanwhile, Stat3 was reported to play a vital role in the innate immune response, anti-inflammatory and repair of damaged tissues [13-15]. Although the bone formation was known to be affected by Stat3, the effect of osteoblast-derived Stat3 on bone remodeling in inflammatory lesions is yet to study.

Hence this study aimed to investigate the effect of Stat3 on the osteoblast-mediated bone remodeling in the inflammatory lesion. The conditional knockout of Stat3 in osteoblasts (Stat3 CKO) was generated via the Cre-loxP recombination system using Osterix-Cre (Osx-Cre) transgenic mice [16-18]. Furthermore, the lipopolysaccharide (LPS) -induced calvarial bone lesions were established on both Stat3 CKO and wildtype mice. With the depletion of Stat3 from osteoblasts, the bone remodeling in inflammatory microenvironments was assessed in vivo and in vitro.

\section{Materials And Methods}

\section{Mouse lines}

The mice were purchased from the Jackson Laboratory (Osx-GFP-Cre, stock no. 006361 and Stat $3^{\mathrm{f} / \mathrm{f}}$, stock no. 016923) $[19,20]$. To generate the mice of Stat3 conditional knockout in osteoblasts (Stat3 $\mathrm{CKO}$ ), the Stat $3^{\mathrm{f} / \mathrm{f}}$ mice were first crossed with the Osx-GFP-Cre transgenic mice to obtain the Osx-GFP-Cre; Stat ${ }^{f /+}$ mice. Then, the Osx-GFP-Cre; Stat ${ }^{f /+}$ mice were crossed with the Stat $3^{f / f}$ mice to get the OsxGFP-Cre; Stat $3^{\mathrm{f} / \mathrm{f}}$ (i.e. Stat3 CKO) mice. Genotyping was conducted by polymerase chain reaction (PCR) of tail lysate. Primer sequences were provided in Table 1. 
For temporal deletion of Stat3 in osteoblasts, the doxycycline administration in drinking water $(0.2 \mathrm{~g}$ doxycycline per liter water; Sigma Aldrich, St Louis, MO) was applied on the Stat3 CKO mice (i.e. to tet-off the function of conditional knockout by Osx-GFP-Cre) until the age of 3-weeks (2 weeks before the establishment of inflammatory calvarial lesions). With the least influence of Stat3-deficiency on the development of animal skeleton, the in vivo knockout of Stat3 in osteoblasts was implemented during the period of inflammatory lesion establishment and sample harvest. For in vitro culture of osteoblasts, the Stat3 CKO mice were raised without doxycycline administration. The animals were used according to guidelines with approval by the Animal Ethical and Welfare Committee of Sun Yat-sen University (approval number SYSU-IACUC-2020-000069).

\section{Establishment of inflammatory calvarial osteolytic lesions}

To study the effects of osteoblastic Stat 3 on bone remodeling in inflammatory microenvironments, the LPS-induced calvaria osteolytic model was established on both Stat3 CKO and the littermate (the control) mice as described in literature [21, 22]. In brief, the LPS (Sigma-Aldrich, St. Louis, MO, USA, L2880, 25 $\mathrm{mg} / \mathrm{kg}$ ) or PBS (sham group) were injected subcutaneously into the tissue pocket surrounding the calvaria and near the midline of the skull between the ears and eyes in 5-week mice. After the injection for 3,7 and 14 days, the animals ( $n=3$ per group) were sacrificed respectively to collect the calvaria. The timeline of doxycycline administration, inflammatory calvaria osteolysis establishment and sample harvest was displayed in Figure 1.

\section{Micro-CT scanning and analysis}

The calvaria were fixed in $4 \%$ para-formaldehyde at $4{ }^{\circ} \mathrm{C}$ overnight, stored in $70 \%$ ethanol for $24 \mathrm{~h}$ and analyzed using a high-resolution micro-CT (Scanco, Brüttisellen, Switzerland) instrument. The scanning protocol was set at an isometric resolution of $15 \mathrm{~mm}$, with X-ray energy settings of $70 \mathrm{kV}$ and $114 \mu \mathrm{A}$. After reconstruction, a square region of interest ( $\mathrm{ROI})$ of the calvaria was selected for further quantitative analysis, including bone volume fraction (BV/TV), trabecular thickness (Tb.Th.), trabecular number (Tb.N.) and trabecular separation (Tb.Sp.). The calvaria were reconstructed into 3-dimensional images using Mimics software (Medical Version 17.0; Materialise, Leuven, Belgium).

\section{Histological staining}

After fixed in $4 \%$ para-formaldehyde at $4^{\circ} \mathrm{C}$ overnight, the calvaria were decalcified in $10 \%$ ethylenediaminetetraacetic acid for 2 weeks, and dehydrated through a series of alcohols and were embedded in paraffin. Paraffin-embedded specimens were sectioned through the coronal plane at a thickness of $5 \mu \mathrm{m}$. Histological analysis was performed as reported previously [23, 24]. The sections were prepared for hematoxylin and eosin (HE) staining and Masson staining, then analyzed under a microscope. To observe the osteoclast activity, the tartrate-resistant acid phosphatase (TRAP) staining was performed using a TRAP kit (kit no. 387A; Sigma-Aldrich, St Louis, MO) according to the manufacturer's instructions. Osteoclasts were identified morphologically as the large multinucleated cells 
within the resorption lacunae. For quantitative analysis, the number of TRAP-positive multinucleated osteoclasts of bone surface area were counted.

\section{Immunohistochemistry staining}

Immunohistochemistry (IHC) of Stat3, runt related transcription factor 2 (Runx2), osteopontin (OPN), alpha-1 type I collagen (COL1A1) were performed to visualize the spatial expression of these proteins in paraffin-embedded sections from calvaria samples. All sections were stained using the HRP-DAB staining kit (CTS017; R\&D Systems, Minneapolis, MN) according to the manufacturer's instructions. The primary antibodies were listed in Table 2. The counterstaining was performed with hematoxylin. The negative controls were treated with phosphate-buffered saline rather than the primary antibodies.

\section{Cell culture and mineralization induction}

Primary osteoblasts were harvested as described previouslyv[25, 26]. Calvaria were collected from 2 to 6day-old Stat3 CKO and wildtype mice, subject to digestion with collagenase type I $(1 \mathrm{mg} / \mathrm{ml})$ in a-minimal essential medium (a-MEM, Gibco) at $37^{\circ} \mathrm{C}$ for 1.5 hours. Then, the cells were collected by centrifugation and seeded into culture dishes in a-MEM containing 10\% fetal bovine serum (FBS, Gibco) and 1\% penicillin/streptomycin (P/S, Gibco). In this study, the osteoblasts from passage $2-3$ were plated at $2 \times 10^{4}$ cells $/ \mathrm{cm}^{2}$ in 6-well plates and cultured in $2 \mathrm{ml}$ of alpha-MEM containing $10 \% \mathrm{FBS}$. Upon reaching approximately $60-70 \%$ confluence, cells were switched to the mineralization induction medium, which is consisted of alpha-MEM supplemented with $10 \%$ (vol/vol) FBS, $10^{-7} \mathrm{M}$ dexamethasone (Sigma, USA), 10 $\mathrm{mM} \beta$-glycerol phosphate (Solarbio, Beijing, China), $50 \mu \mathrm{M}$ ascorbate-2-phosphate (Solarbio, Beijing, China). During the mineralization induction, the cells were co-cultured either with $1 \mu \mathrm{g} / \mathrm{ml}$ LPS (SigmaAldrich, USA) for inflammatory stimulation [27], or with PBS as the control.

\section{Alizarin red staining and calcium deposition determination}

After mineralization induction for 21 days, the cells were gently washed three times with PBS, fixed in $4 \%$ paraformaldehyde for $20 \mathrm{~min}$, and stained with alizarin red S solution for $15 \mathrm{~min}$. Images of extracellular matrix mineralization nodules were obtained using an inverted microscope with a digital camera. For quantitative analysis, the mineralization nodules were extracted using $10 \%$ cetylpyridinium chloride. The absorbance was measured at a wavelength of $540 \mathrm{~nm}$ on a spectrophotometer (Thermo Fisher Scientific).

\section{Cell proliferation assay}

A 5-ethynyl-20-deoxyuridine (EdU) assay kit (Beyotime Biotechnology, Shanghai, China) was applied to assess the proliferation of cells. Cells were seeded into a 6-well plate with density of $2 \times 10^{5}$ cells per well. When the cells reached $60-70 \%$ confluence, they were incubated with EdU buffer at $37^{\circ} \mathrm{C}$ for $2 \mathrm{~h}$, fixed with $4 \%$ formaldehyde for $0.5 \mathrm{~h}$ and permeabilized with $0.1 \%$ Triton $\mathrm{X}-100$ for $20 \mathrm{~min}$. EdU staining solution was added into the plate followed by the staining of nuclei with Hoechst. Fluorescence microscopy was performed in five randomly selected fields with an inverted microscope (Axio Observer Z1, Zeiss, Oberkochen, Germany) to analyze the proliferation rates. 


\section{Transwell migration assay}

Transwell plates ( $8 \mu \mathrm{m}$ pore size; Costar, Corning, NY, USA) were used to perform the cell migration assay according to the manufacture's protocol. Briefly, a total of $100 \mu$ l of a serum-free suspension $2.5 \times 10^{5}$ cells $/ \mathrm{ml}$ was seeded in the upper membrane of inserts in the presence of LPS ( 0 and $1 \mu \mathrm{g} / \mathrm{ml})$ while $600 \mu \mathrm{l}$ of medium with $10 \%$ serum was added in the lower chamber. After incubation for $12 \mathrm{~h}$ at $37^{\circ} \mathrm{C}$, the cells remaining at the upper surface of the membrane were removed with cotton swabs, and the cells on the lower surface of the membrane were considered as the migrated cells. Migrated cells were fixed in $4 \%$ paraformaldehyde for $20 \mathrm{~min}$ and stained with crystal violet for $30 \mathrm{~min}$ at room temperature. The cells in five random microscopy fields were counted using an inverted microscope (Axio Observer Z1, Zeiss, Oberkochen, Germany).

\section{RNA isolation, reverse transcription, and qRT-PCR analysis}

Total RNA of mouse calvarial and osteoblasts were extracted using a RNA extraction kit (Esunbio, Shanghai, China) according to the manufacturer's instructions and reverse transcribed using a PrimeScript ${ }^{\mathrm{TM}} \mathrm{RT}$ Master Mix (Takara Bio, Ohtsu, Japan). The quantification was performed on a Light Cycler 480(Roche, Basel, Switzerland) with the TB Green ${ }^{\mathrm{TM}}$ Premix Ex Taq ${ }^{\mathrm{TM}}$ II reagent (Takara Bio, Ohtsu, Japan). The relative expression of target gene was normalized in relative to the level of GAPDH using the $2^{-\triangle \Delta C T}$ method. Primer sequences were displayed in the Table 1.

\section{Western blot}

Total protein of mouse calvaria and osteoblasts lysates were extracted using the RIPA lysis buffer (CWBIOTECH, Beijing, China) with protease (CWBIOTECH, Beijing, China) and phosphatase inhibitors (CWBIOTECH, Beijing, China) according to the manufacturer's instructions. The proteins were separated by sodium dodecyl sulfate-polyacrylamide gel electrophoresis (SDS-PAGE, Genscript, Nanjing, China) and transferred to a polyvinylidene fluoride (PVDF) membrane (Millipore, Bedford, MA, USA). The membrane was then blocked with $5 \%$ bovine serum albumin (BSA, Biofroxx, Germany) for $1.5 \mathrm{~h}$, incubated with relevant primary antibodies (Table 2 ) at $4^{\circ} \mathrm{C}$ overnight, and then incubated with corresponding secondary antibodies for $1 \mathrm{~h}$ at room temperature. Bands were detected with a chemiluminescence kit (Millipore, Bedford, MA, USA).

\section{Statistical analysis}

All animal studies were performed using gender- and age-matched animals, with the wild-type littermates as controls (CON). Data were derived from multiple independent experiments from distinct mice or cell culture plates. The data were presented as means \pm standard deviation (SD). Student's t-tests were used to compare the difference between Stat3 CKO and CON. The GraphPad Prism 7.0 software was applied for statistical analysis. The significance level was set at $P<0.05$.

\section{Results}




\section{Characterization of Stat3 CKO mice}

To achieve the conditional knockout of Stat3 in osteoblast, the transgenic mice of Osx-Cre was used to cross with Stat $3^{\mathrm{f} / \mathrm{f}}$ mice. In Fig. 2A, the mouse genotypes, including Osx-Cre; Stat $3^{\mathrm{f} / \mathrm{f}}$ (Stat3 CKO) and Cre negative littermate control, were displayed. The calvaria isolated from 5-week-old Stat3 CKO mice showed positive GFP signal due to Osx-GFP-Cre, whereas it was absent in CON mice (Fig. $2 B$ ). To verify the elimination of Stat3 in osteoblasts, the quantitative real-time polymerase chain reaction (qRT-PCR) and western blot were performed to assess the expression of Stat3. Stat3 were found in the osteoblasts in the calvaria of the 5-week-old Con mice, but were hardly expressed in the calvaria of the Stat3 CKO mice (Fig. 2C, D) $\left({ }^{\star *} P<0.01\right)$.

\section{Depletion of Stat3 in osteoblasts led to a decrease of bone mass in calvarial osteolytic lesions}

To explore the impact of Stat3-deficiency on bone regeneration in inflammatory microenvironment, the LPS-stimulated calvaria lesion was induced on both Stat3 CKO and control mice. According to the morphological observation of calvaria from day 3 to day 14 (Fig. 3A), the knockout of Stat3 in osteoblasts led to an increase of LPS-induced osteolysis, which was characterized by visible osteolysis damage and bone surface erosion, especially at day 3 and day 7. Micro-CT 3D reconstruction observations indicated an obvious bone loss in the Stat3 CKO mice on calvaria at day 7 (Fig. 3B). Meanwhile, no significant difference of microstructural indices between CON and Stat3 CKO mice was observed at day 3 and 14. On the other hand, a marked reduction of trabecular bone mass in Stat3 CKO mice was found at day 7 , assessed by the CKO vs. CON groups of BV/TV, Tb.Th., Tb.N., and Tb.Sp. (Fig. 3C) $\left({ }^{\star} P<0.05, \star \star P<0.01\right)$.

\section{Stat3 deficiency in osteoblasts caused an increased inflammatory osteolysis}

Considering that the obvious lesion difference between CON and Stat3 CKO mice was found at day 7 after LPS-stimulation, these samples were collected for paraffin section preparation. According to HE staining, the inflammatory cell infiltration along with significant bone erosion was observed in the calvaria of Stat3 CKO mice (Fig. 4A). The decrease of mineralized bone matrix in Stat3 CKO mice was confirmed by Masson staining (Fig. 4B) $(* \star P<0.01)$. Consistent with the increased bone erosion, the osteolytic lesion with abundant TRAP-positive osteoclasts was found on Stat3 CKO mice, whereas the CON group was presented with fewer TRAP-positive osteoclasts (Fig. 4C) ( $\left.{ }^{\star \star} P<0.01\right)$. Moreover, the increased mRNA expression of RANKL in osteolytic lesion was found on Stat3 CKO group (Fig. 4D) (vs. $\left.\mathrm{CON},{ }^{\star} P<0.05\right)$.

\section{In the bone destructive lesion, the osteogenic markers were decreased in Stat3 CKO mice}

The IHC staining observations revealed that the number of Stat3 positive osteoblasts in Stat3 CKO group was dramatically dropped compared with CON group ( $\left.{ }^{* \star} P<0.001\right)$ (Fig. $\left.5 \mathrm{~A}\right)$. Meanwhile, the numbers of Runx2, OPN and COL1A1 positive osteoblasts per hpf in osteolytic lesion were significantly decreased in Stats3 CKO mice ( $\left.{ }^{*} P<0.01\right)$ (Fig. 5A). A considerably reduction of the mRNA and protein expressions 
of osteogenic markers Runx2, OPN, and COL1A1 were detected in the calvaria of Stat3 CKO mice, compared with CON group ( $* P<0.05, \star \star P<0.01)$ (Fig. 5B-D).

\section{Knockdown of Stat3 in osteoblasts led to a reduced mineralization in the inflammatory microenvironment in vitro}

To gain insight into the Stat3-mediated bone matrix mineralization in osteoblasts, osteoblasts from primary cell culture of passage 2-3 were seeded into plates followed by mineralization induction for 21 days. Less formation of calcium deposit was observed in the Stat3-dificient cells when compared with the Con, which was visualized by the Alizarin Red staining ( $\left.{ }^{\star \star \star} P<0.001\right)$ (Fig. 6A).

The expression of total Stat3 and nuclear phosphorylated Stat3 (p-Stat3) was significantly decreased in Stat3 CKO cells in both normal and inflammatory microenvironment, confirming that Stat3 gene was effectively knocked out in osteoblasts of Stat3 CKO mice ( ${ }^{\star} P<0.05$, $\left.{ }^{\star *} * P<0.001\right)$ (Fig. 6B-C). The lack of

Stat3 significantly suppressed the mRNA expression of Runx2, COL $1 A 1$, and $O P N$ in osteoblasts $\left({ }^{\star} P<0.05\right.$, $\left.{ }^{\star} * P<0.01,{ }^{*} * * P<0.001\right)$ (Fig. $\left.6 \mathrm{~B}-\mathrm{C}\right)$ ). The mRNA expression of $R A N K L$ in osteoblasts was increased while difference was not found in the expression of $O P G$, which was consistent with the in vivo data $\left({ }^{\star} P<0.05\right)$ (Fig. 6D-E). The protein levels of osteogenic markers were also down-regulated in both normal and inflammatory microenvironment, compared with the control ( $\left.{ }^{\star} P<0.05,{ }^{\star *} P<0.01\right)$ (Fig. 5F-G).

\section{The proliferation and migration of Stat3-dificient osteoblasts were inhibited in the inflammatory microenvironment}

According to the EdU assay, fewer EdU positive cells were observed in Stat3 CKO group in both normal and inflammatory microenvironment $(* * P<0.01)$ (Fig. 7A, B). The number of migrated osteoblasts in the Stat3 CKO group was significantly decreased in normal microenvironment and further less in inflammatory microenvironment, compared with the control ( $\left.{ }^{\star \star *} P<0.001\right)$ (Fig. $\left.7 \mathrm{C}, \mathrm{D}\right)$.

\section{Discussion}

Bone remodeling in inflammatory bone destructive lesion has always been an important clinical research topic in stomatology. To explore how Stat3 regulates osteoblast-mediated bone remodeling in the inflammatory lesion, we cultivated osteoblast-specific Stat3 knockout transgenic mice (Stat3 CKO) and established LPS-induced inflammatory bone destructive lesion on both Stat3 CKO and wildtype mice. Osteoblasts from the calvaria of neonatal mice were isolated and cultured in vitro to investigate the effect of Stat3 deficiency on the ossification, signals transduction in osteoclast activation, proliferation and migration of osteoblasts in inflammatory microenvironments. Our findings indicated that Stat 3 plays a promoting role in bone regeneration and remodeling mediated by osteoblasts in inflammatory lesion.

Germline Stat3 depletion-induced early embryonic lethality was observed in 1997 [28]. The impaired contractility and metabolic homeostasis by Stat3 deficiency was found in cardiomyocyte-targeted Stat3 knockout (KO) mice [29]. During the forced swim, tail suspension and open-field tests, antidepressive-like 
behavior in microglia-specific Stat3 KO mice proved that microglial Stat3 regulates depression-related behaviors via neuronal M-CSF-mediated synaptic activity [30]. Collectively, Stat3 is widely expressed in different tissues and cells, participates in the regulation of many physiological functions including proliferation, differentiation, growth, apoptosis and inflammation [31]. In this study, we used Tet-Off system in Osx-Cre mice to control the expression of Stat3 in osteoblasts in time for doxycycline can reversibly inhibit the expression of Cre-GFP fusion protein and prevent the activation of CKO. The purpose of withdrawing doxycycline before 3 weeks of age is to ensure the bone development was not affected and bone mass between two groups was similar. So it could avoid errors caused by their own development defects and body size in further research. After next two weeks of metabolism, the original osteoblast-derived Stat3 would be consumed by apoptosis, then Stat3 would be conditionally knocked out in the newborn osteoblasts.

LPS from the cell membrane of gram-negative bacteria is recognized as the main pathogenic factor positively related to infectious bone destruction. A recent literature has shown that LPS inhibited bone remodeling and promoted oxidative stress accumulation in alveolar bone lesions [32]. The hypothesis that factors within the inflamed joint impede bone formation at arthritic sites by inhibiting osteoblast maturation was supported by less mineralized bone formed at bone surfaces adjacent to inflammation, compared to surfaces adjacent to normal bone marrow [33]. These studies raise the interesting question as to how inflammation impairs osteoblast maturation and function. Bandow $\mathrm{K}$ et al. subsequently demonstrated that the inhibitory effect of LPS on osteoblast differentiation is Myd88-dependent: LPS suppresses the mRNA expression of Runx2, osterix (Osx), and activating transcription factor 4 (ATF4) during osteoblast differentiation in the wild-type, but not in the myd88 flox/flox osteoblasts [34]. Pretreatment with JNK inhibitor not only attenuated both of phosphorylation-JNK and ERK1/2 enhanced by LPS in MC3T3-E1 cells, but also reversed the down-regulated expressions of osteoblast-specific genes including ALP and BSP induced by LPS, reaching to the conclusion that LPS could inhibit osteoblast differentiation via activation of JNK pathway [35]. It has also been reported that LPS can suppress osteoblastic differentiation by affecting multiple pathways, such as BMP/Smad signaling, Wnt/ $\beta$-catenin signaling, and Notch signaling [36-38]. As was shown in this study, the depletion of Stat3 in osteoblasts resulted in a decline of regenerated bone mass at the bone-repair site in accordance with micro-CT analysis. Our data from Masson staining also indicated that bone fraction was reduced in the lesion of Stat3 CKO mice. Given the findings that the lack of Stat3 led to less regenerated bone, we also found that Stat3 could affect bone matrix mineralization function of osteoblasts in vitro. Moreover, the protein and mRNA expressions of osteogenic markers Runx2, OPN and COL1A1 were diminished in Stat3 CKO group, further confirming the role of Stat3 as a positive regulator of bone regeneration in osteoblasts. Taken together, our findings revealed that loss of Stat3 delays bone remodeling in mice with impaired osteoblast bone formation activity.

LPS-induced overproduction of inflammatory such as tumor necrosis factor- $a$ (TNF-a), interleukin-1 $\beta$ (IL$1 \beta)$ and RANKL [39-42] contributes to most inflammatory response that are involved in the pathogenesis of inflammation-stimulated oral bone diseases [43]. The role of inflammation has been closely 
associated with bone loss and osteoclastogenesis [44]. Among infection-induced inflammation, the components of invading bacteria (e.g. LPS) will activate macrophages. Factors secreted from activated macrophages increase sphingosine kinase 1 (SPHK1) activity in bone marrow stromal cells (BMSCs), resulting in the overproduction of sphingosine-1-phosphate (S1P). Then S1P acts on its receptor sphingosine-1-phosphate receptor 1 (S1PR1) in BMSCs, which results in the overproduction of RANKL and eventually induces bone resorption via the activation of RANK in osteoclast precursors [45]. During the active phase of rheumatoid arthritis, the inflamed synovium comprising T-cells, synovial fibroblasts, and macrophages, produces inflammatory cytokines and RANKL which can activate osteoclasts and lead to bone destruction [46]. RANKL/RANK/OPG is an important pathway modulated by osteoblasts which affect the bone mass density via the regulation of osteoblasts and osteoclasts functions [47]. A closer look at the osteoclast activity via TRAP staining showed that osteoclast number of Stat3 CKO mice was significantly greater in our study. Besides, Stat3 deficiency in osteoblasts promoted LPS-induced osteoclastogenesis mediated by RANKL expression was found both in vivo and in vitro. A possible explanation might be that, in the inflammatory environment, Stat3 not only plays a role in ossification of osteoblasts, but also enhances osteoclast activity indirectly by regulating osteoclast-related signal molecules secreted by osteoblasts. But its underlying mechanism still needs further investigation.

Existing studies have suggested Stat3 can induce the expression of proliferative, anti-apoptotic, angiogenic and metastatic genes that promote tumor development and growth, such as B-cell lymphomaextra large (Bcl-xL), B-cell lymphoma-2,Bcl-2 (Bcl-2), Cyclin D1, c-Myc, vascular endothelial growth factor (VEGF) and HIF1a [48-51]. Sim and other scholars have shown that gp130-Stat1/3 signaling pathway plays a key role in stimulating chondrocyte proliferation [52]; under hypoxia, interleukin-8 (IL-8) enhances the proliferation of bone marrow mesenchymal stem cells (BMSCs) through Akt-Stat3 pathway and reduces the apoptosis rate of cells [53]. Recent work from Yu X et al. reported that hypoxia can enhance BMSCs migration through Stat3 signal transduction, and up-regulated gene expression related to cell migration, such as matrix metalloproteinase 7 (MMP7), matrix metalloproteinase 9 (MMP9) and C-X-C chemokine receptor type 4 (CXCR4) [54]. In the present study, the notion that the proliferation and migration ability of osteoblasts are inhibited owing to Stat3 deficiency and inflammation is supported by decreased migration cells number and EdU positive cells number of Stat3 CKO group in vitro. This may explain the underlying mechanism of inflammation and Stat3 deficiency leading to changes in osteoblast mineralization. In brief, osteoblast-derived Stat3 is associated with pro-proliferative, anti-inflammatory and osteogenic growth factor activities during bone remodeling.

Taken together, our study indicated Stat3 plays an important role in osteoblast-mediated bone remodeling in inflammatory microenvironment. Loss of Stat3 in osteoblasts caused decreased bone regeneration in inflammatory microenvironment, which might be resulted from the effect of Stat3 on the ossification, signals transduction in osteoclast activation, proliferation and migration of osteoblasts. Based on these results, Stat3 could serve as a promising treatment target for inflammatory bone diseases.

\section{Abbreviations}


Stat3: signal transducer and activator of transcription 3; CKO: conditional knockout; PCR: polymerase chain reaction; IHC: immunohistochemistry; FBS: fetal bovine serum; BSA: bovine serum albumin; Osx: osterix; OPN: osteopontin; COL1A1: collagen l; Runx2: runt-related transcription factor-2

\section{Declarations}

\section{Acknowledgements}

Not applicable.

\section{Authors' Contributions}

JYF, ZJH and XLZ designed the experiments; JYF, ZJH, LTC, JRL,XF, LZL and ZWH performed experiments; JYF and ZJH analyzed the data; JYF wrote the manuscript; XLZ participated in critical revision of the paper. All authors read and approved the final manuscript. All authors discussed the results and approved the final manuscript.

\section{Founding}

This work was supported by the National Natural Science Foundation of China (Grant No. 81470731).

\section{Availability of data and materials}

All datasets used and/or analyzed during the current study are available from the corresponding author on reasonable request.

\section{Ethics approval and consent to participate}

Mice were used according to federal guidelines and as approved by the Animal Ethical and Welfare Committee of Sun Yat-sen University (approval number SYSU-IACUC-2020-000069).

\section{Consent for publication}

Not applicable.

\section{Competing interests}

The authors declare that they have no competing interests.

\section{References}

1. Xing Q, Ye Q, Fan $M$, et al. Porphyromonas gingivalis lipopolysaccharide inhibits the osteoblastic differentiation of preosteoblasts by activating Notch1 signaling. J Cell Physiol. 2010;225(1):106-14.

2. Ikeda K, Takeshita S. The role of osteoclast differentiation and function in skeletal homeostasis. J Biochem. 2016 Jan;159(1):1-8. 
3. Ralston SH, De Crombrugghe B. Genetic regulation of bone mass and susceptibility to osteoporosis. Genes Dev. 2006;20(18):2492-506.

4. Buettner R, Mora LB, Jove R. Activated STAT signaling in human tumors provides novel molecular targets for therapeutic intervention. Clin Cancer Res. 2002 Apr;8(4):945-54.

5. Nadiminty N, Lou W, Lee SO, Lin X, Trump DL, Gao AC. Stat3 activation of NF-\{kappa\}B p100 processing involves CBP/p300-mediated acetylation. Proc Natl Acad Sci. 2006 May;9(19):7264-9. 103(.

6. Li J. JAK-STAT and bone metabolism. JAKSTAT. 2013 Jul;1(3):e23930. 2(.

7. Davis SD, Schaller J, Wedgwood RJ. Job's Syndrome. Recurrent, "cold", staphylococcal abscesses. Lancet. 1966;287:1013-5.

8. Grimbacher B, Puck JM, Holland SM. Hyper-IgE Recurrent Infections Syndrome. In: Ochs HD, Smith CIE, Puck JM, editors. Primary Immunodeficiency Diseases: A Molecular \& Cellular Approach. New York City: Oxford University Press, Inc.; 2007. pp. 496-504.

9. Grimbacher B, Holland SM, Gallin JI, Greenberg F, Hill SC, Malech HL, et al. Hyper-lgE syndrome with recurrent infections-an autosomal dominant multisystem disorder. N Engl J Med. 1999;340:692702.

10. Huang Z, Feng J, Feng $X$, et al. Loss of signal transducer and activator of transcription 3 impaired the osteogenesis of mesenchymal progenitor cells in vivo and in vitro. Cell Biosci. 2021;11(1):172.

11. Lei L, Huang Z, Feng J, et al. Loss of receptor tyrosine kinase-like orphan receptor 2 impairs the osteogenesis of mBMSCs by inhibiting signal transducer and activator of transcription 3. Stem Cell Res Ther. 2020;11(1):137.

12. Yang Y, Chung MR, Zhou S, et al. Stat3 controls osteoclast differentiation and bone homeostasis by regulating NFATc1 transcription. J Biol Chem. 2019;294(42):15395-407.

13. Galoczova M, Coates P, Vojtesek B. Stat3, stem cells, cancer stem cells and p63. Cell Mol Biol Lett. 2018;23:12.

14. Samavati L, Rastogi R, Du W, et al. Stat3 tyrosine phosphorylation is critical for interleukin 1 beta and interleukin-6 production in response to lipopolysaccharide and live bacteria. Mol Immunol. 2009 May;46(8-9):1867-77.

15. Kortylewski M, Kujawski M, Herrmann A, et al. Toll-like receptor 9 activation of signal transducer and activator of transcription 3 constrains its agonist-based immunotherapy. Cancer Res. 2009 Mar 15;69(6):2497-505.

16. Liu F, Woitge HW, Braut A, Kronenberg MS, Lichtler AC, Mina M, Kream BE. Expression and activity of osteoblast-targeted Cre recombinase transgenes in murine skeletal tissues. Int J Dev Biol. 2004;48:645-53.

17. Rodda SJ, McMahon AP. Distinct roles for Hedgehog and canonical Wnt signaling in specification, differentiation and maintenance of osteoblast progenitors. Development. 2006;133:3231-44. 
18. Dacquin R, Starbuck M, Schinke T, Karsenty G. Mouse alpha1(I)-collagen promoter is the best known promoter to drive efficient Cre recombinase expression in osteoblast. Dev Dyn. 2002;224:245-51.

19. Logan M, Martin JF, Nagy A, Lobe C, Olson EN, Tabin CJ. Expression of Cre recombinase in the developing mouse limb bud driven by a Prxl enhancer. Genesis. 2002;33:77-80.

20. Rodda SJ, McMahon AP. Distinct roles for Hedgehog and canonical Wnt signaling in specification, differentiation and maintenance of osteoblast progenitors. Development. 2006 Aug;133(16):323144.

21. Liu Z, Yuan X, Liu M, Fernandes G, Zhang Y, Yang S, lonita CN, Yang S. Antimicrobial Peptide Combined with BMP2-Modified Mesenchymal Stem Cells Promotes Calvarial Repair in an Osteolytic Model. Mol Ther. 2018 Jan 3;26(1):199-207.

22. Yu X, Quan J, Long W, Chen H, Wang R, Guo J, Lin X, Mai S. LL-37 inhibits LPS-induced inflammation and stimulates the osteogenic differentiation of BMSCs via P2X7 receptor and MAPK signaling pathway. Exp Cell Res. 2018 Nov 15;372(2):178-187.

23. Tao YW, Xing Q, Li D, et al. Association between Increased Inducible Costimulator/Inducible Costimulator Ligand Expression with Bone Destruction in Apical Periodontitis. J Endod. 2019 Jul;45(7):890-7.

24. Hu K, Olsen BR. Osteoblast-derived VEGF regulates osteoblast differentiation and bone formation during bone repair. J Clin Invest. 2016 Feb;126(2):509-26.

25. Yagishita N, Yamamoto Y, Yoshizawa T, et al. Aberrant growth plate development in VDR/RXR gamma double null mutant mice. Endocrinology. 2001 Dec;142(12):5332-41.

26. Doolittle ML, Ackert-Bicknell CL, Jonason JH. Isolation and Culture of Neonatal Mouse Calvarial Osteoblasts. Methods Mol Biol. 2021;2230:425-36.

27. Zhang Y, Gu X, Li D, et al. METTL3 Regulates Osteoblast Differentiation and Inflammatory Response via Smad Signaling and MAPK Signaling. Int J Mol Sci. 2019;21(1):199.

28. Takeda K, Noguchi K, Shi W, et al. Targeted disruption of the mouse Stat3 gene leads to early embryonic lethality. Proc Natl Acad Sci USA. 1997 Apr;15(8):3801-4. 94(.

29. Altara R, Harmancey R, Didion SP, et al. Cardiac STAT3 Deficiency Impairs Contractility and Metabolic Homeostasis in Hypertension. Front Pharmacol. 2016;7:436.

30. Kwon SH, Han JK, Choi M, Kwon YJ, Kim SJ, et al. Dysfunction of Microglial STAT3 Alleviates Depressive Behavior via Neuron-Microglia Interactions. Neuropsychopharmacology. 2017 Sep;42(10):2072-86.

31. Corry KA, Zhou H, Brustovetsky T, et al. Stat3 in osteocytes mediates osteogenic response to loading. Bone Rep. 2019;11:100218.

32. Zhao Y, Gao J, Zhang Y, Gan X, Yu H. Cyclosporine A Promotes Bone Remodeling in LPS-Related Inflammation via Inhibiting ROS/ERK Signaling: Studies In Vivo and In Vitro. Oxid Med Cell Longev. 2021 Jan 7;2021:8836599. 
33. Walsh NC, Reinwald S, Manning CA, et al. Osteoblast function is compromised at sites of focal bone erosion in inflammatory arthritis. J Bone Miner Res. 2009 Sep;24(9):1572-85.

34. Bandow K, Maeda A, Kakimoto K, et al. Molecular mechanisms of the inhibitory effect of lipopolysaccharide (LPS) on osteoblast differentiation. Biochem Biophys Res Commun. 2010 Nov 26;402(4):755-61.

35. Guo C, Yuan L, Wang JG, et al. Lipopolysaccharide (LPS) induces the apoptosis and inhibits osteoblast differentiation through JNK pathway in MC3T3-E1 cells. Inflammation. 2014 Apr;37(2):621-31.

36. Daigang L, Jining Q, Jinlai L, et al. LPS-stimulated inflammation inhibits BMP-9-induced osteoblastic differentiation through crosstalk between BMP/MAPK and Smad signaling. Exp Cell Res. 2016 Feb 1;341(1):54-60.

37. Xing $Q, Y e Q$, Fan $M$, et al. Porphyromonas gingivalis lipopolysaccharide inhibits the osteoblastic differentiation of preosteoblasts by activating Notch1 signaling. J Cell Physiol. 2010 Oct;225(1):106-14.

38. Tang Y, Zhou X, Gao B, et al. Modulation of Wnt/ $\beta$-catenin signaling attenuates periapical bone lesions. J Dent Res. 2014 Feb;93(2):175-82.

39. Boyle WJ, Simonet WS, Lacey DL. Osteoclast differentiation and activation. Nature. 2003 May 15;423(6937):337-42.

40. Lee YM, Fujikado N, Manaka H,et al. IL-1 plays an important role in the bone metabolism under physiological conditions. Int Immunol. 2010;22:805-16.

41. Yoshitake F, Itoh S, Narita H, Ishihara K, Ebisu S. Interleukin-6 directly inhibits osteoclast differentiation by suppressing receptor activator of NF-kappaB signaling pathways. J Biol Chem. 2008;283:11535-40.

42. Atanga E, Dolder S, Dauwalder T, et al. TNFalpha inhibits the development of osteoclasts through osteoblast-derived GM-CSF. Bone. 2011;49:1090-100.

43. Siqueira JF Jr, Rocas IN. Bacterial pathogenesis and mediators in apical periodontitis. Braz Dent J. 2007;18(4):267-80.

44. Adamopoulos IE. Inflammation in bone physiology and pathology. Curr Opin Rheumatol. 2018;30(1):59-64.

45. Xiao L, Zhou Y, Zhu L, et al. SPHK1-S1PR1-RANKL Axis Regulates the Interactions Between Macrophages and BMSCs in Inflammatory Bone Loss. J Bone Miner Res. 2018 Jun;33(6):1090-104.

46. Tateiwa D, Yoshikawa H, Kaito T. Cartilage and Bone Destruction in Arthritis: Pathogenesis and Treatment Strategy: A Literature Review. Cells. 2019;8(8):818.

47. Mitchell BD, Yerges-Armstrong LM. The genetics of bone loss: challenges and prospects. J Clin Endocrinol Metab. 2011 May;96(5):1258-68.

48. Fan Y, Mao R, Yang J. NF-kappaB and STAT3 signaling pathways collaboratively link inflammation to cancer. Protein Cell. 2013;4:176-85. 
49. Bromberg J. Stat proteins and oncogenesis. J Clin Invest. 2002 May;109(9):1139-42.

50. Sumimoto $H$, Imabayashi F, Iwata T, Kawakami Y. The BRAF-MAPK signaling pathway is essential for cancer-immune evasion in human melanoma cells. J Exp Med. 2006 Jul 10;203(7):1651-6.

51. Wang T, Niu G, Kortylewski M, et al. Regulation of the innate and adaptive immune responses by Stat-3 signaling in tumor cells. Nat Med. 2004 Jan;10(1):48-54.

52. Sims NA, Jenkins BJ, Quinn JM, et al. Glycoprotein 130 regulates bone turnover and bone size by distinct downstream signaling pathways. J Clin Invest. 2004 Feb;113(3):379-89.

53. Shen L, Zhang S, Zhang X, et al. [Enhancing the ability of autophagy and proliferation of bone marrow mesenchymal stem cells by interleukin-8 through Akt-Stat3 pathway in hypoxic environment]. Sheng Wu Gong Cheng Xue Bao. 2016 Oct 25;32(10):1422-1432.

54. Yu X, Wan Q, Cheng G, et al. CoCl2, a mimic of hypoxia, enhances bone marrow mesenchymal stem cells migration and osteogenic differentiation via STAT3 signaling pathway. Cell Biol Int. 2018 Sep;42(10):1321-9.

\section{Tables}

\section{Tablel Primers for PCR and qRT-PCR}

\begin{tabular}{llll}
\hline Gene & Forward & Reverse & Application \\
\hline Osx & GAGAATAGGAACTTCGGAATAGTAAC & CCCTGGAAGTGACTAGCATTG & PCR \\
Stat3 & TTG ACC TGT GCT CCT ACA AAA A & CCC TAG ATT AGG CCA GCA CA & PCR \\
GAPDH & CCTTCCGTGTTCCTACCC & CAACCTGGTCCTCAGTGTAG & qRT-PCR \\
Stat3 & CATCCTGAAGCTGACCCAGG & TATTGCTGCAGGTCGTTGGT & qRT-PCR \\
Runx2 & CCGTCACCTCCATCCTCTTTC & AATACGCATCACAACAGCCACA & qRT-PCR \\
COL 1 A1 & CCCAAGGAAAAGAAGCACGTC & ACATTAGGCGCAGGAAGGTCA & qRT-PCR \\
OPN & CTCCAATCGTCCCTACAGTCG & CCAAGCTATCACCTCGGCC & qRT-PCR \\
OPG & TTACCTGGAGATCGAATTCTGCTTG & GTGCTTTCGATGAAGTCTCACCTG & qRT-PCR \\
RANKL & AAACTGGTCGGGCAATTCTG & AGGGTTGGACACCTGAATGCTA & qRT-PCR \\
\hline
\end{tabular}


Table2 Antibodies used for immunoblotting

\begin{tabular}{lll}
\hline \multicolumn{1}{c}{ Marker (species) } & \multicolumn{1}{c}{ Dilution } & \multicolumn{1}{c}{ Distributor/source (catalog number) } \\
\hline Primary antibody & $1: 1000(\mathrm{WB})$ & ZEN BIO (380437) \\
\hline OPN rabbit pAb & $1: 1000$ (WB) & AB clonal (A16891) \\
COL1A1 goat pAb & $1: 1000(\mathrm{WB})$ & CST (9139) \\
Stat3 mouse mAb & $1: 2000(\mathrm{WB})$ & CST (4113) \\
pStat3 mouse mAb & $1: 1000(\mathrm{WB})$ & CST (8486) \\
Runx2 rabbit mAb & $1: 100(\mathrm{IHC})$ & CST (9139) \\
Stat3 mouse mAb & $1: 200(\mathrm{IHC})$ & ZEN BIO (380437) \\
OPN rabbit $\mathrm{pAb}$ & $1: 100(\mathrm{IHC})$ & CST (8486) \\
Runx2 rabbit mAb & $1: 100(\mathrm{IHC})$ & AB clonal (A16891) \\
COL1A1 goat $\mathrm{pAb}$ & $1: 2000(\mathrm{WB})$ & CST (7076) \\
\hline Secondary antibody & & CST (7074) \\
\hline Anti-mouse IgG HRP-linked Ab & &
\end{tabular}

\section{Figures}

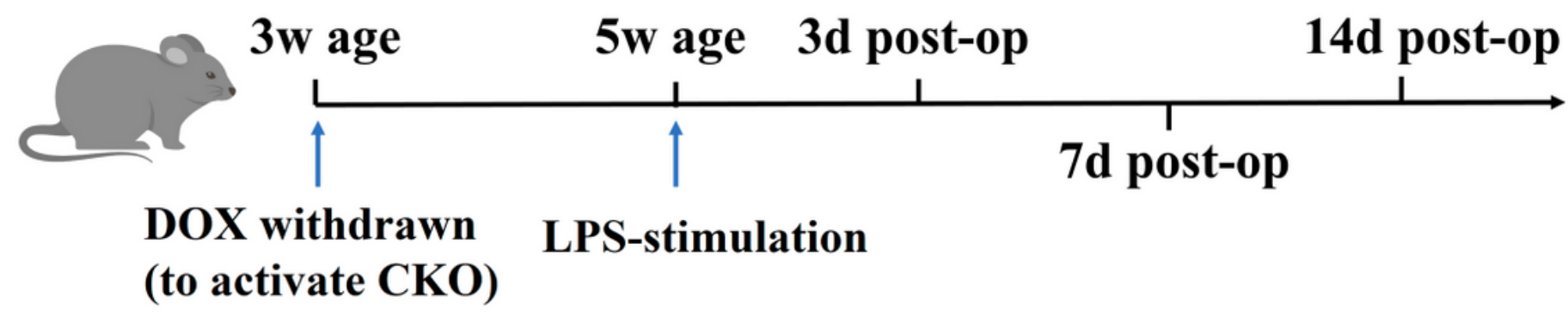


Figure 1

The process of establishing inflammatory bone destruction model in murine calvaria. For temporal deletion of Stat3 in osteoblasts, the doxycycline administration in drinking water was applied on the Stat3 CKO mice until the age of 3-weeks (2 weeks before the establishment of inflammatory calvarial lesions). The LPS or PBS (sham group) were injected subcutaneously into the tissue pocket surrounding the calvaria and near the midline of the skull between the ears and eyes in 5-week-old Stat3 CKO and the littermate (the control) mice. After the injection for 3,7 and 14 days, the animals ( $n=3$ per group) were sacrificed respectively to collect the calvaria.

A

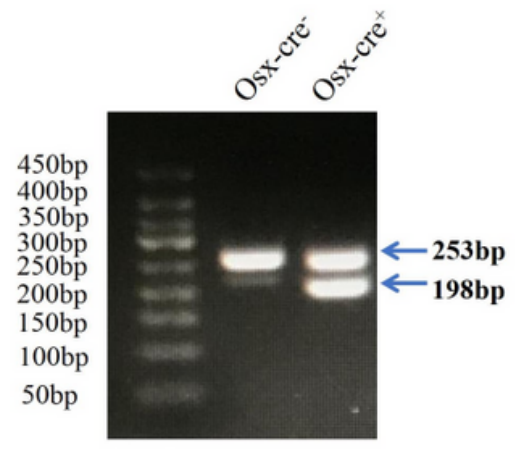

C

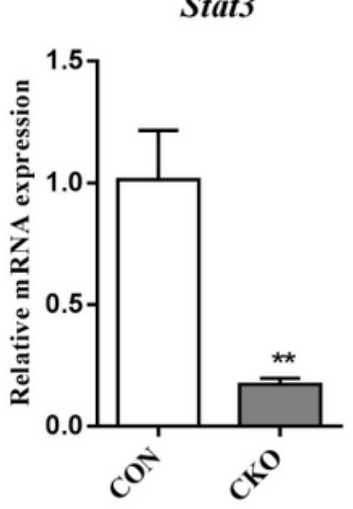

B

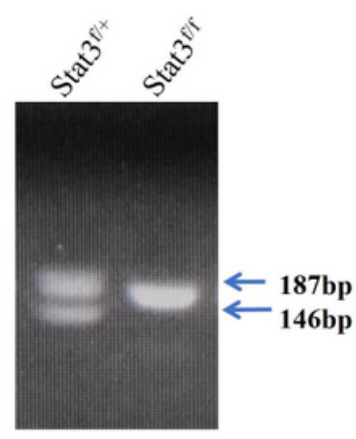

GFP

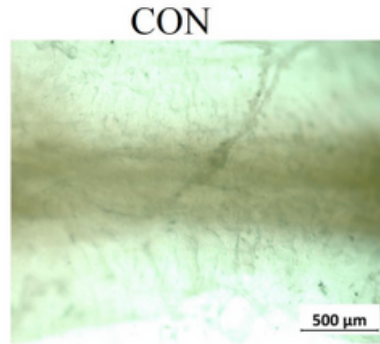

CKO
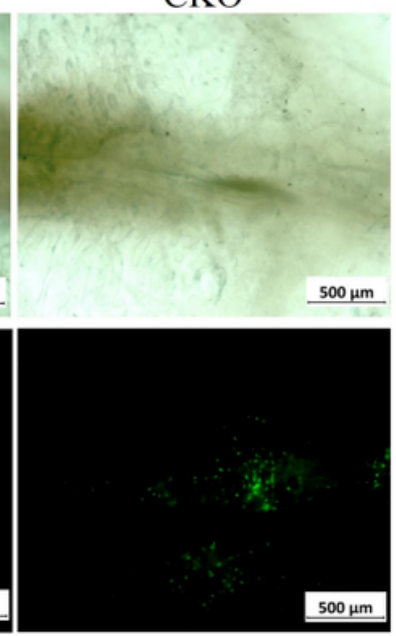

\section{D}

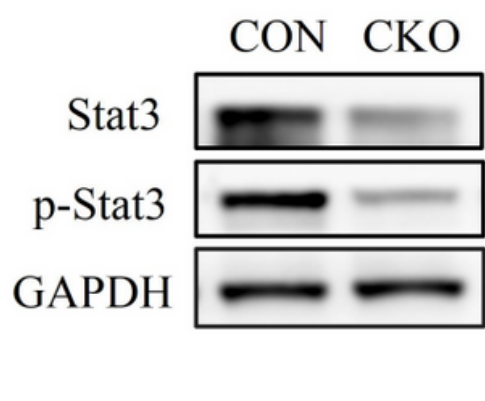

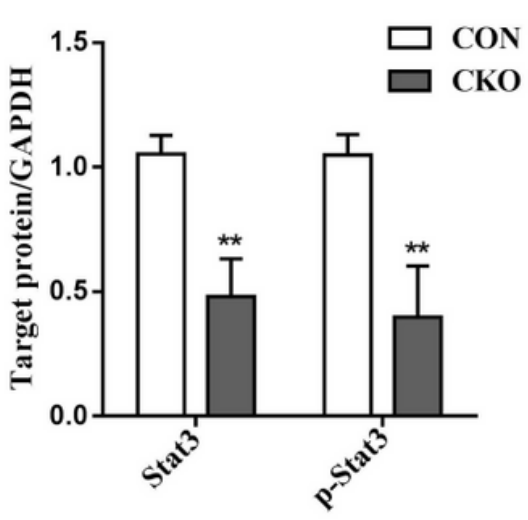

Figure 2

Characterization of the Stat3 CKO mice. A. Genotyping of Osx-Cre ; Stat $3 f / f$ mice and Stat $3 f /+$ mice tail DNA. DNA ladder: 50bp. B. Bright field and immunoflurescent images of calvaria from CON mice and Stat3 CKO mice in the absence of doxycycline. Scale bar: $500 \mu \mathrm{m}$. C. the mRNA expression of Stat3 in the calvaria from CON mice and Stat3 CKO mice in the absence of doxycycline. $n=3,{ }^{*} P<0.01$. D. Western blot and quantitative analysis results of Stat3 and p-Stat3 in the calvaria from CON mice and Stat3 CKO mice in the absence of doxycycline. $n=4,{ }^{*} P<0.01$ v.s. $C O N$. 
A

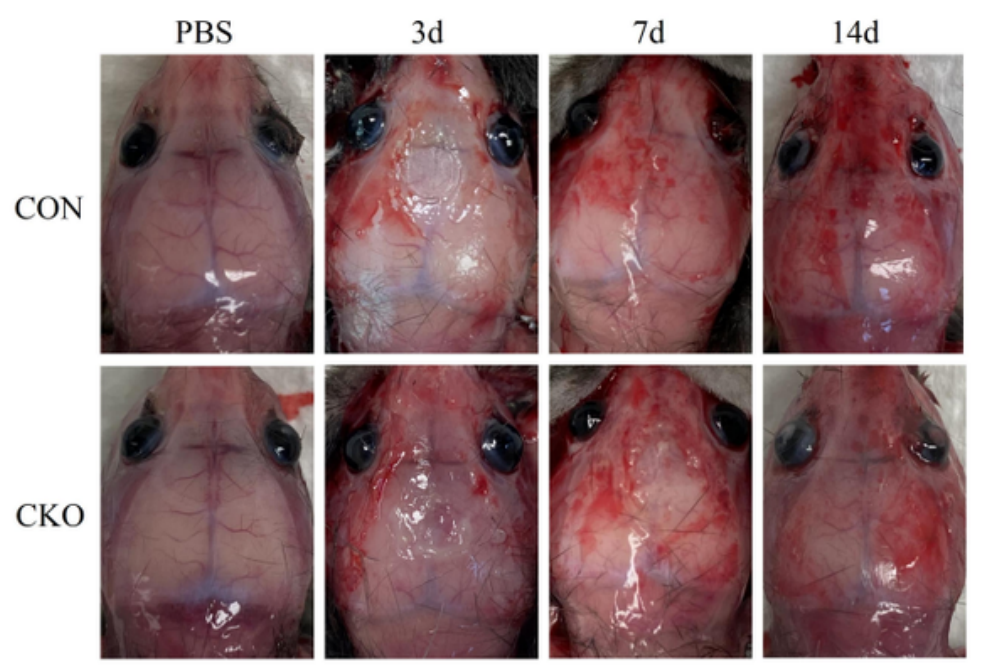

B
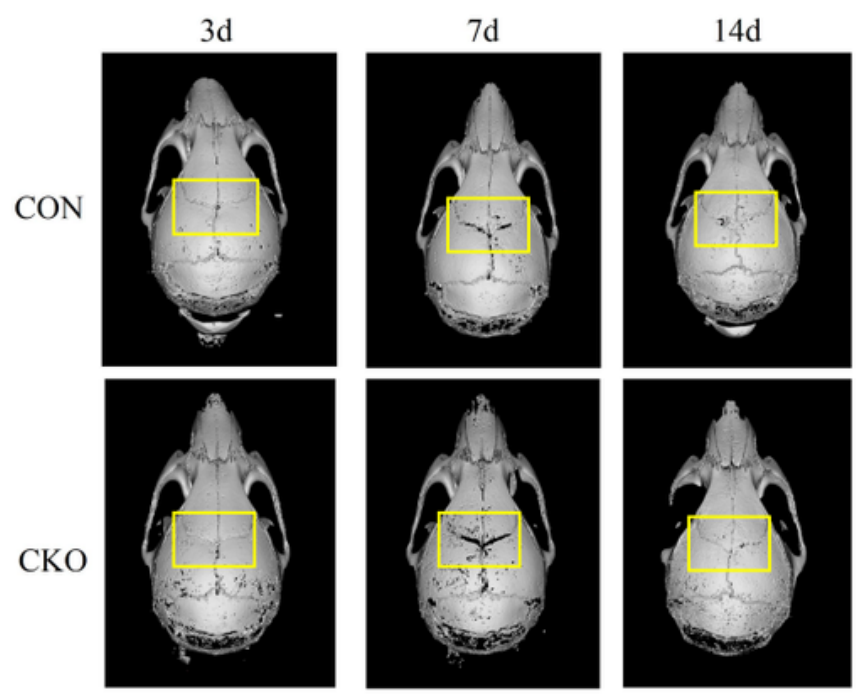

C

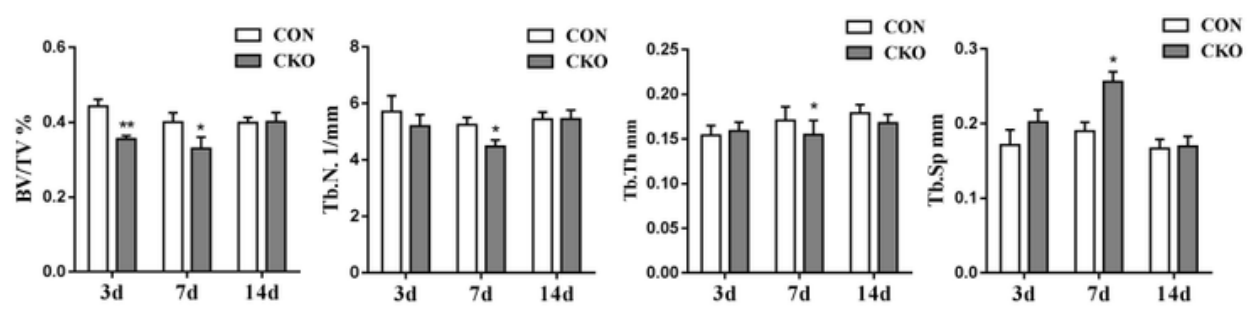

\section{Figure 3}

Depletion of Stat3 in osteoblasts led to decreased bone mass in calvarial osteolytic lesion. A. Morphological observation of calvaria from day 3 to day 14. B. Representative micro-CT threedimensional reconstruction images of calvaria from day 3 to day 14. C. Microstructural indices including bone volume/tissue volume (BV/TV), trabecular separation (Tb.Sp), trabecular number (Tb.N), and trabecular thickness (Tb.Th) were analyzed. $n=6,{ }^{*}<<0.05, * * P<0.01$ v.s. CON. 

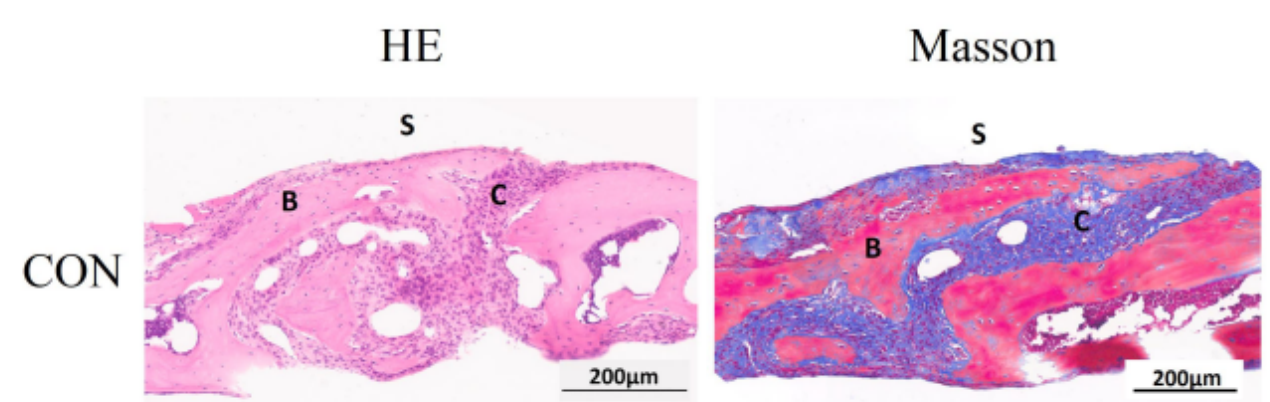

S
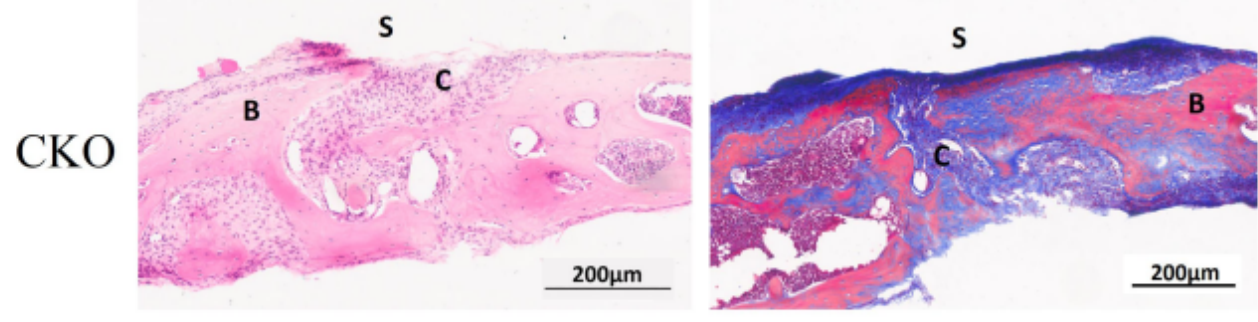

B

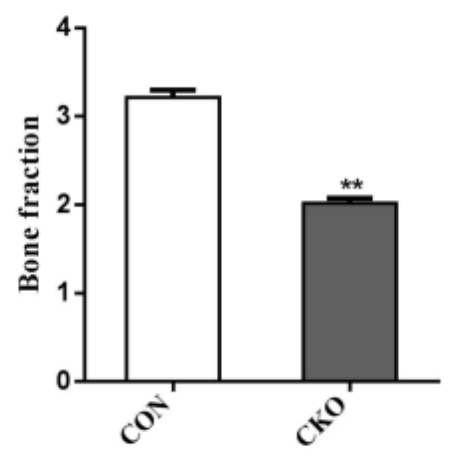

C

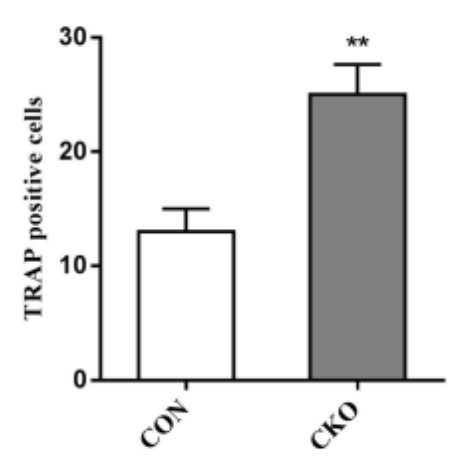

TRAP

S

C

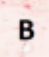

B

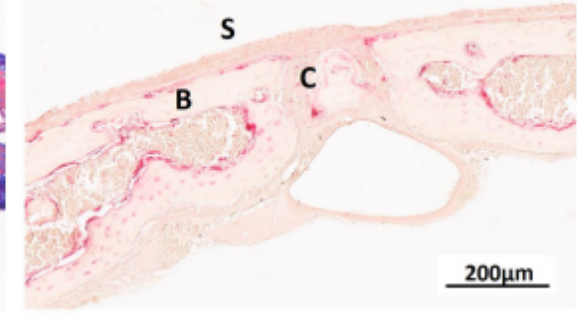

\section{Figure 4}

Osteoclast activity was increased in LPS-induced osteolysis lesion in Stat3 CKO mice. A. The calvaria sections of day 7 were stained with HE, Masson and TRAP staining. Apparent inflammatory cell infiltration along with significant bone erosion was observed in calvaria of Stat3 CKO mice in HE staining. The collagen fibers were blue and the bone matrix was red in Masson staining. Representative TRAP staining histological sections of calvaria from CON and Stat3 CKO mice at day 7 were shown. Scale bar: $200 \mu \mathrm{m}$. B. More bone fraction in CON mice was presented in Masson staining. $n=3, * * P<0.01$. (C) Quantitative result of TRAP positive osteoclasts number per hpf. $n=3, \star \star P<0.01$. B-bone, C-connective tissue, S-surface. (D)The results of qRT-PCR revealed a significant decrease of mRNA expression of RANKL and OPG in Stat3 CKO mice compared with CON mice. $n=3,{ }^{\star} P<0.05$ v.s. CON. 
$\mathbf{A}$

Stat3
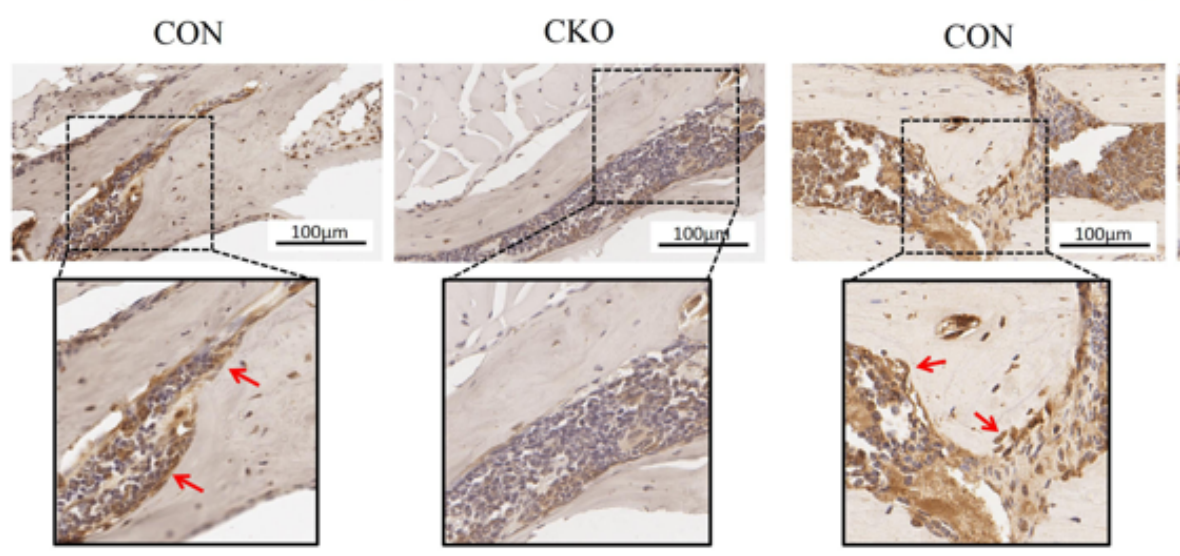

OPN

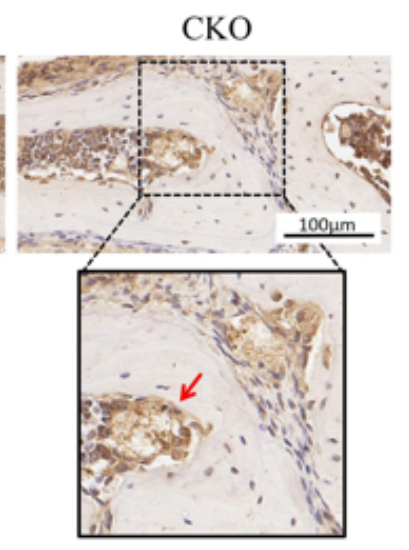

Runx2
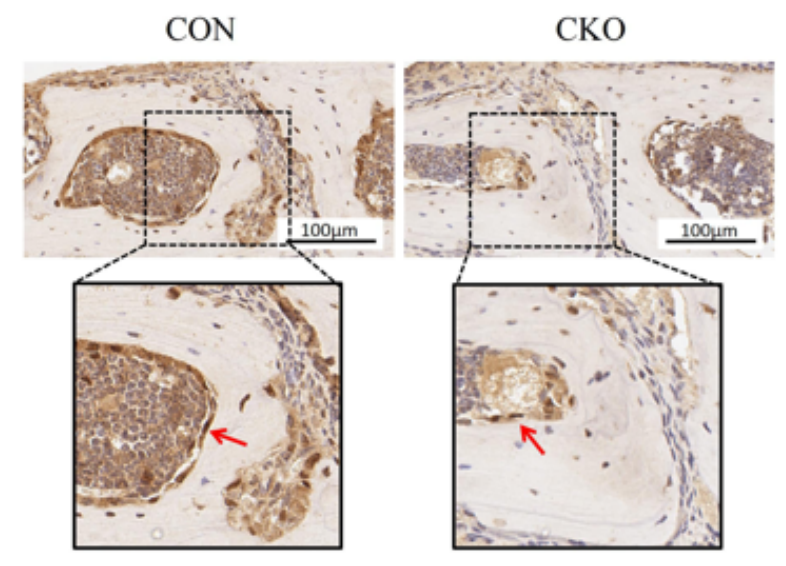

COL1A1
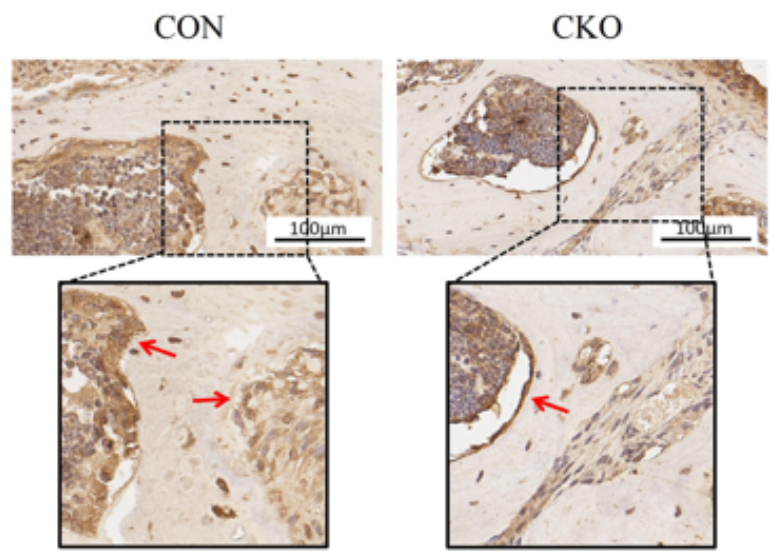

B

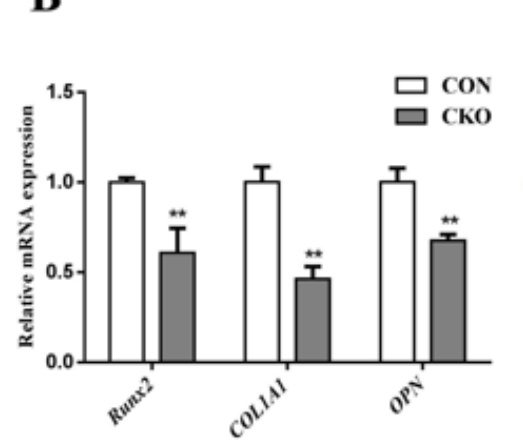

C

CON CKO

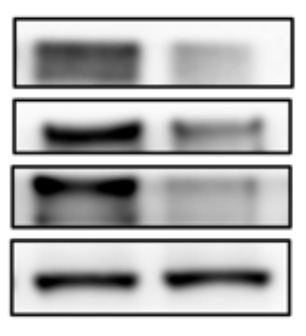

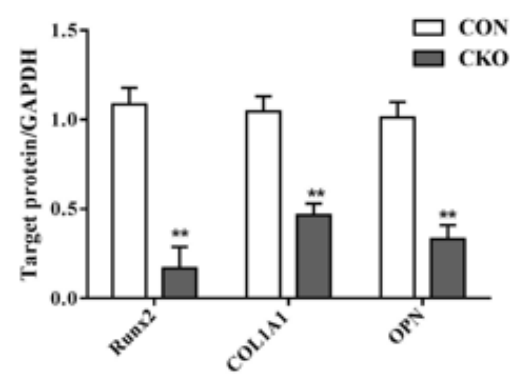

\section{Figure 5}

Stat3 CKO mice expressed less osteogenic markers in inflammatory bone destruction lesion. A. Immunohistochemistry (IHC) for Stat3, OPN, Runx2 and COL1A1 in calvaria sections of day 7 showed that Stat3 was knocked down in the osteoblasts in calvaria of Stat3 CKO mice while Stat3 CKO mice clearly exhibited decreased positive cells of OPN, Runx2 and COL1A1 compared with CON mice. Red arrows indicate positive cells. Scale bar: $100 \mu \mathrm{m}$. B. The mRNA expressions of Runx2, OPN and COL1A1 in 
Stat3 CKO mice were significantly decreased compared with CON mice. $n=3,{ }^{*} \mathrm{P}<0.01$ vs. CON. C. Western blot results of the osteogenic proteins in calvaria from CON mice and Stat3 CKO mice were shown. $n=4,{ }^{* *} \mathrm{P}<0.01$ v.s. CON.

A
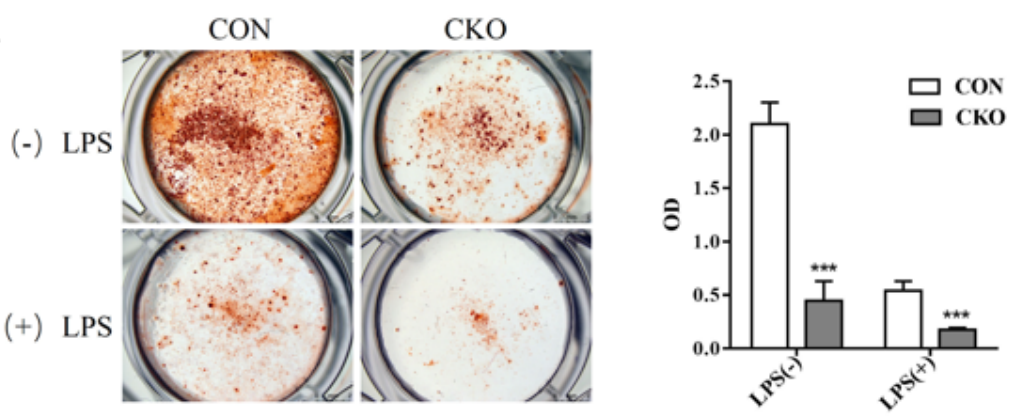

B

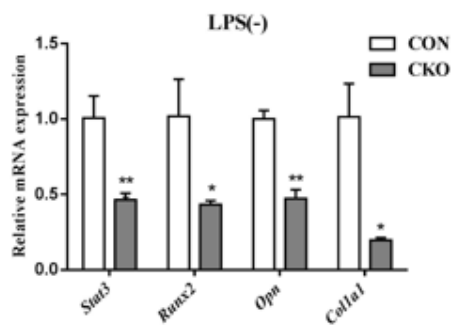

C

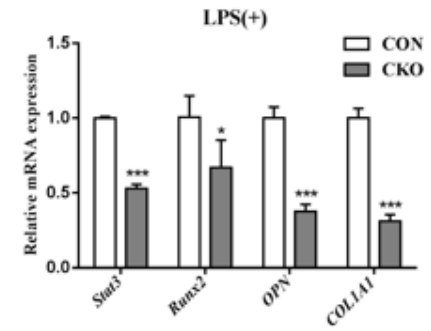

D

$\mathbf{E}$
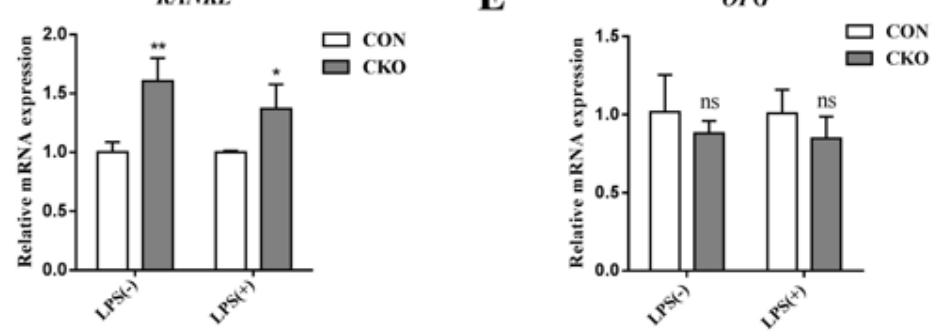

F
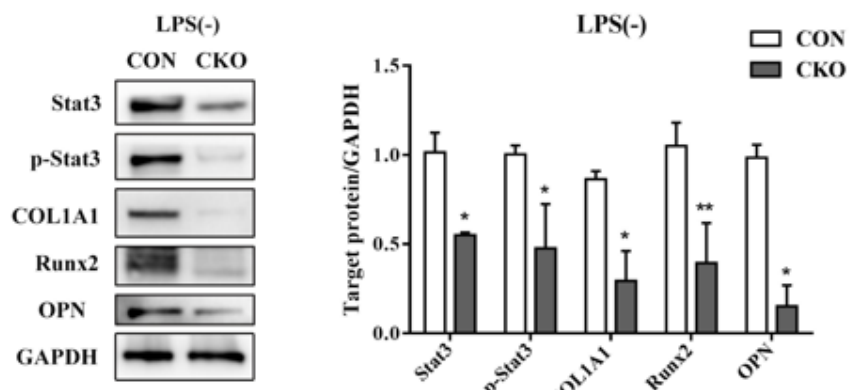

$\mathbf{F}$

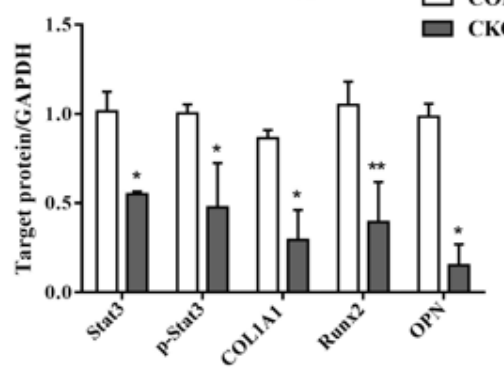

G
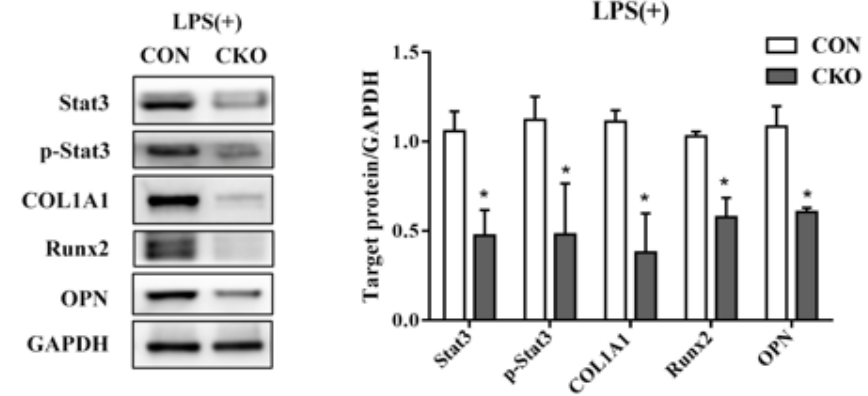

\section{Figure 6}

Deficiency of Stat3 in osteoblasts led to reduced bone matrix mineralization in inflammatory microenvironment. A. Representative alizarin red staining images were shown after mineralization 
induction for 21 days. Alizarin red concentrations were determined by a quantitative procedure using CPC. $n=4,{ }^{* *} P<0.001$ v.s. CON. B-C. Representative results of the mRNA expression of Stat 3 and osteogenic specific markers after mineralization induction for 21 days. $n=3$, ${ }^{\star}{ }^{*} P<0.01$ v.s. CON. D-E. The mRNA expression of RANKL and OPG in Stat3 CKO mice compared with CON mice. $n=3,{ }^{*} P<0.05$ v.s. CON. F-G. The expressions of osteogenic markers were assessed by western blotting. Quantitative analysis of Stat3, $p$-Stat3 and osteogenic markers expression levels. $n=3, * P<0.05$, ${ }^{*} P<0.01$ v.s. CON.

A

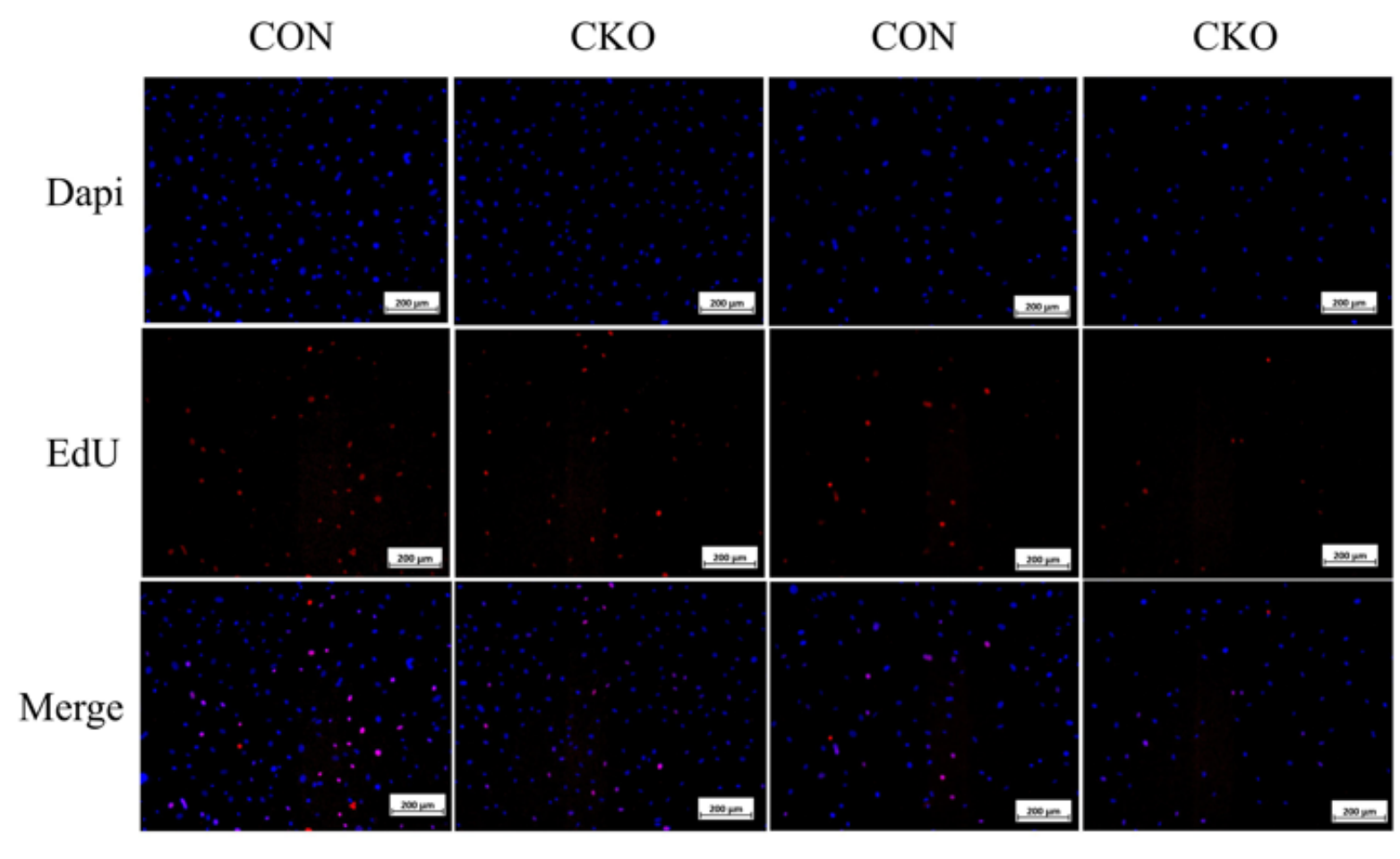

B

D
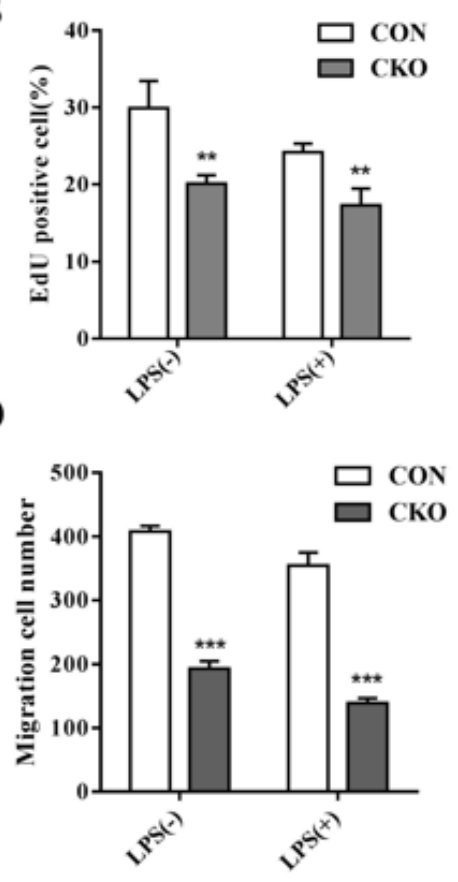

C

CON

CKO

(-) LPS

(+) LPS
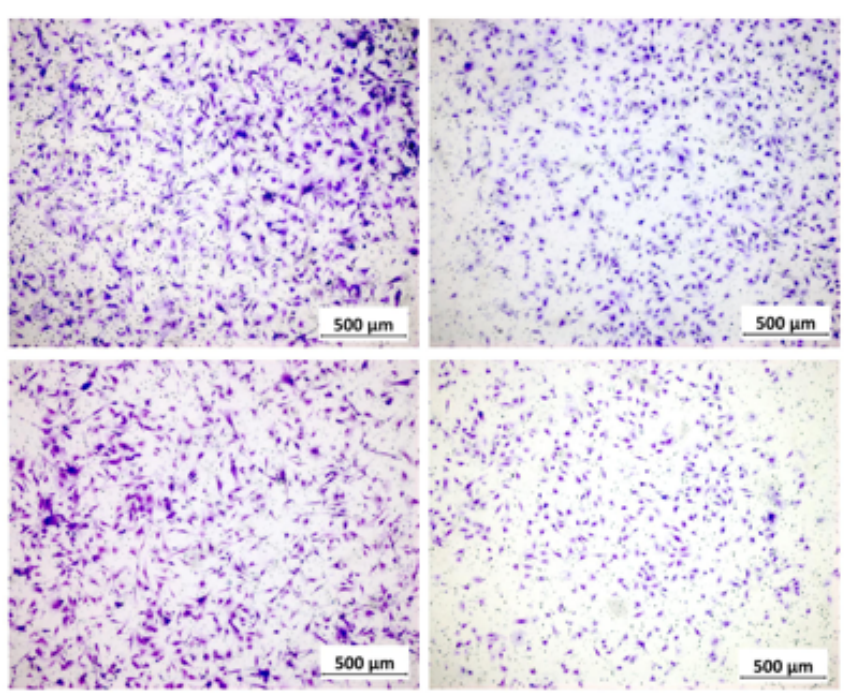

Figure 7 
The proliferation and migration of Stat3-dificient osteoblasts were inhibited in inflammatory microenvironment. A-B. Representative images and quantitative analysis of EdU proliferation assay in CON group and Stat3 CKO group with or without LPS were presented. Blue: Dapi staining of nuclei with all cells; red: staining of EdU with proliferating cells; overlay: the percentage of proliferating cells. Scale bar $=200 \mu \mathrm{m} . n=4,{ }^{\star} P<0.01$ v.s. CON. C-D. Representative images of crystal violet staining of migrated cells and the calculated number of migrated cells in five random microscopy fields were shown. Scale bar $=500 \mu \mathrm{m} . \mathrm{n}=4, \star \star \star \mathrm{P}<0.001$ v.s. $\mathrm{CON}$. 